\title{
What makes a good clinical conversation on physical activity? A Scoping review exploring what is known to inform the development of physical activity resources to support healthcare professionals in routine practice
}

\author{
Hamish Reid ${ }^{1,2}$, Jessica Caterson ${ }^{3}$, Robert Copeland ${ }^{2}$ \\ 1. Moving Medicine, Faculty of Sport and Exercise Medicine, 6 Hill Square, Edinburgh, U.K. \\ 2. Advanced Wellbeing Research Centre, Sheffield Hallam University, Sheffield, United Kingdom \\ 3. Imperial College Healthcare NHS Trust, Praed Street, London, W2 1NY, G.B.
}

\section{Table of Contents}

Introduction.

Aim

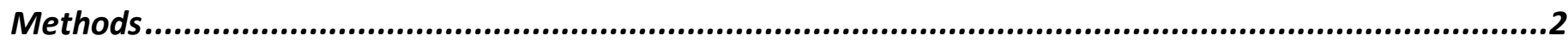

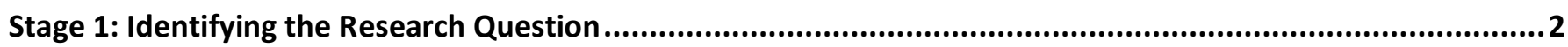

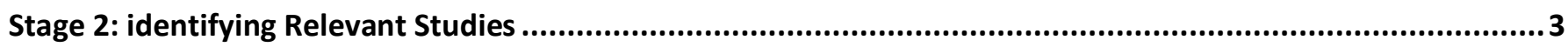

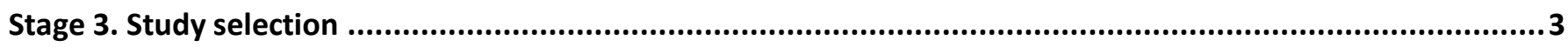

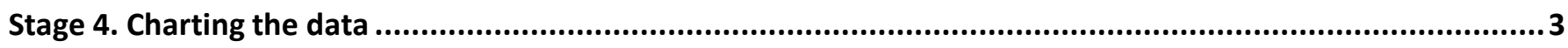

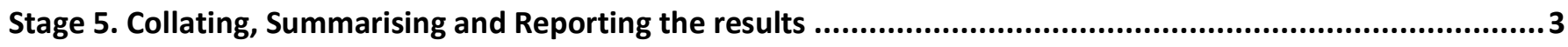

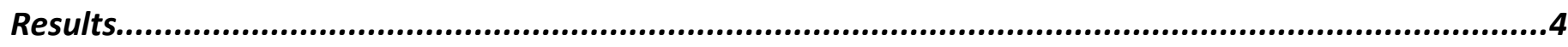

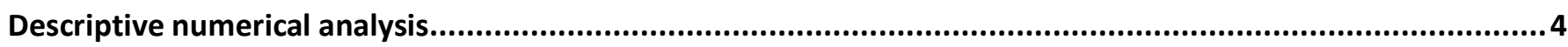

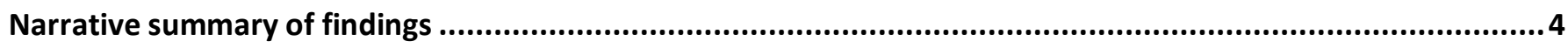

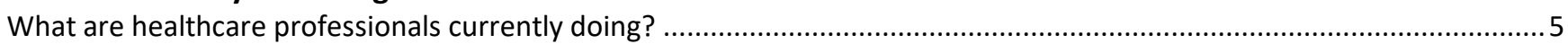

What influences physical activity conversations in clinical practice? ............................................................................. 6

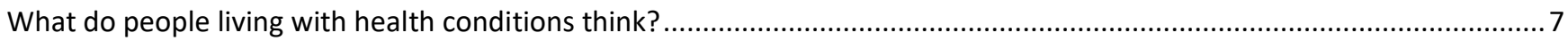

How does previous training impact on conversations?

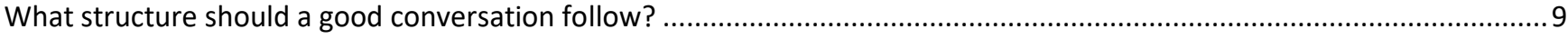

What works when making change happen?

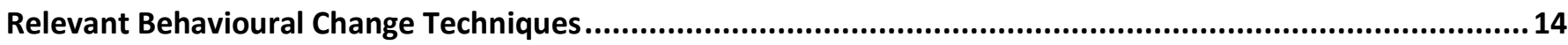

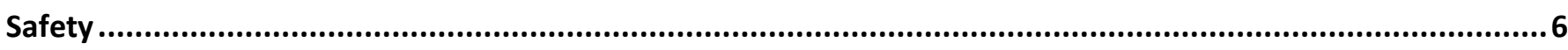

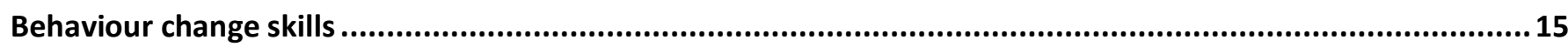

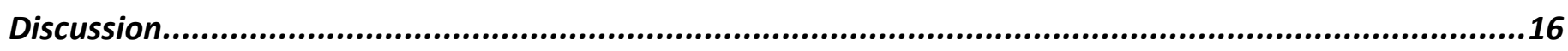

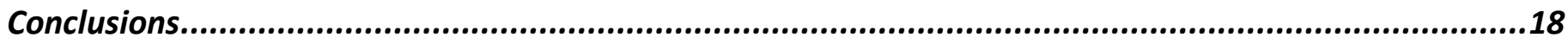

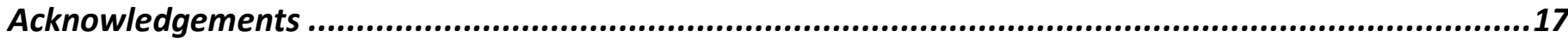

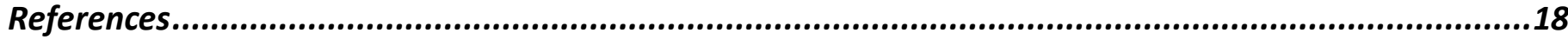




\section{Introduction}

Physical inactivity directly accounts for $6 \%$ of deaths per year globally,[1,2] and is ranked as the $4^{\text {th }}$ most prevalent risk factor for global morbidity and premature mortality by the World Health Organisation (WHO).[1,2]

Promotion of physical activity is therefore a valuable target for improving overall population health. Healthcare systems and the professionals within them form a vital component of population-level intervention.[3-6] Individuals with chronic health conditions are some of the least active in society and notoriously hard to engage in physical activity interventions. Healthcare systems have greater access and involvement with this population, and may therefore may be particularly important in accessing this under-resourced and poorly supported group. $[7,8]$

System-wide approaches in healthcare show promise.[6] In particular, patient-centred conversations between healthcare professionals and people living with chronic conditions can lead to sustained behaviour change.[9-14] However, there is a lack of confidence and awareness of approaches to consultations in physical activity amongst healthcare professionals.[15-24] In addition, the availability of tools or educational platforms to enable healthcare professionals is limited.[20,25]

In 2018 the Faculty of Sport and Exercise Medicine was tasked by Public Health England, as part of the national Moving Health Professionals Programme in England, with developing educational resources around physical activity for healthcare professionals.[6] We undertook this scoping review to understand how we might best design educational resources to support frequent and effective conversations between healthcare professionals and people living with health conditions.

\section{Aim}

This scoping review aims to gain a broad insight into the makeup and delivery of effective physical activity conversations in healthcare. In addition, the results will inform the development of resources to support healthcare professionals having routine conversations on physical activity in their day-to-day practice.

\section{Methods}

We undertook this scoping review according to the 5-stage protocol by Arksey and O'Malley and reported according to the PRISMA extension for scoping reviews checklist (PRISMA-ScR).[26,27]

\section{Stage 1: Identifying the Research Question}

We identified two research questions:

- What is known about the effectiveness and acceptability of physical activity consultations in healthcare?

- What is known about strategies to implement routine physical activity conversations in healthcare? 
We identified studies by:

- Searching electronic databases: Web of Science, TRIP and Ovid (MEDLINE)

- Manually searching reference lists of influential manuscripts

- Reviewing publications by authors emerging as leaders in the field

Our search strategy combined terms around 'healthcare' with those around 'physical activity' and 'behaviour change'. We identified free text, subject heading, and mesh terms by reviewing the terms attributed to relevant papers we were already aware of. OVID search strategy combined mesh terms with keyword searches. TRIP and Web of Science search strategies used keywords with truncation symbols where appropriate. We searched English language publications but didn't limit them by manuscript type. The search was first undertaken in June 2018 and updated in April 2021.

Table 1. Inclusion and Exclusion criteria

\section{Inclusion criteria}

- Research reporting on any healthcare environment

- Research reporting healthcare interactions for either preventative or

therapeutic purposes

- Research reporting physical activity as a discrete intervention

- Research reporting inteventions focussed on changing the consulting

behaviour of clinical staff and healthcare services around physical activity and

behaviour change

- Articles reported in the English language

-Articles reported in peer reviewed and grey literature

- Guidelines and reports from national bodies in the United Kingdom

-All research designs considered

\section{Exclusion criteria}

- Research reporting physical activity as part of a generalised 'lifestyle' intervention

-Articles focussing on specific disease area management

- Research reporting weight loss as the primary outcome

- Opinion articles without a structured approach

\section{Stage 3. Study selection}

H.R. screened titles and abstracts and 10\% verified by J.C. Any conflict was discussed and put to R.C. if not resolved. In addition, H.R. undertook Full-text reviewing and J.C verified $10 \%$.

\section{Stage 4. Charting the data}

Data were extracted and entered into a data charting form in Excel. We recorded author, title, article type, year of publication, region, population, aims, methods, important results, and citations.

\section{Stage 5. Collating, Summarising and Reporting the results}

We present data in two forms as recommended by Arksey and O'Malley [26]: 
1. Fundamental numerical analysis of study characteristics including geographical region, population and empirical/non-empirical approach with the breakdown

2. We then undertook a narrative summary of results, organising results thematically. We developed themes as they emerged from results rather than a priori.

\section{Results}

\section{Descriptive numerical analysis}

We identified 616 references for screening $(n=596$ from databases and $n=20$ from hand searching). Following screening and removal of duplicates, we included 48 studies for analysis. Figure 1 depicts a study selection flowchart.

Figure 1. Study selection flowchart

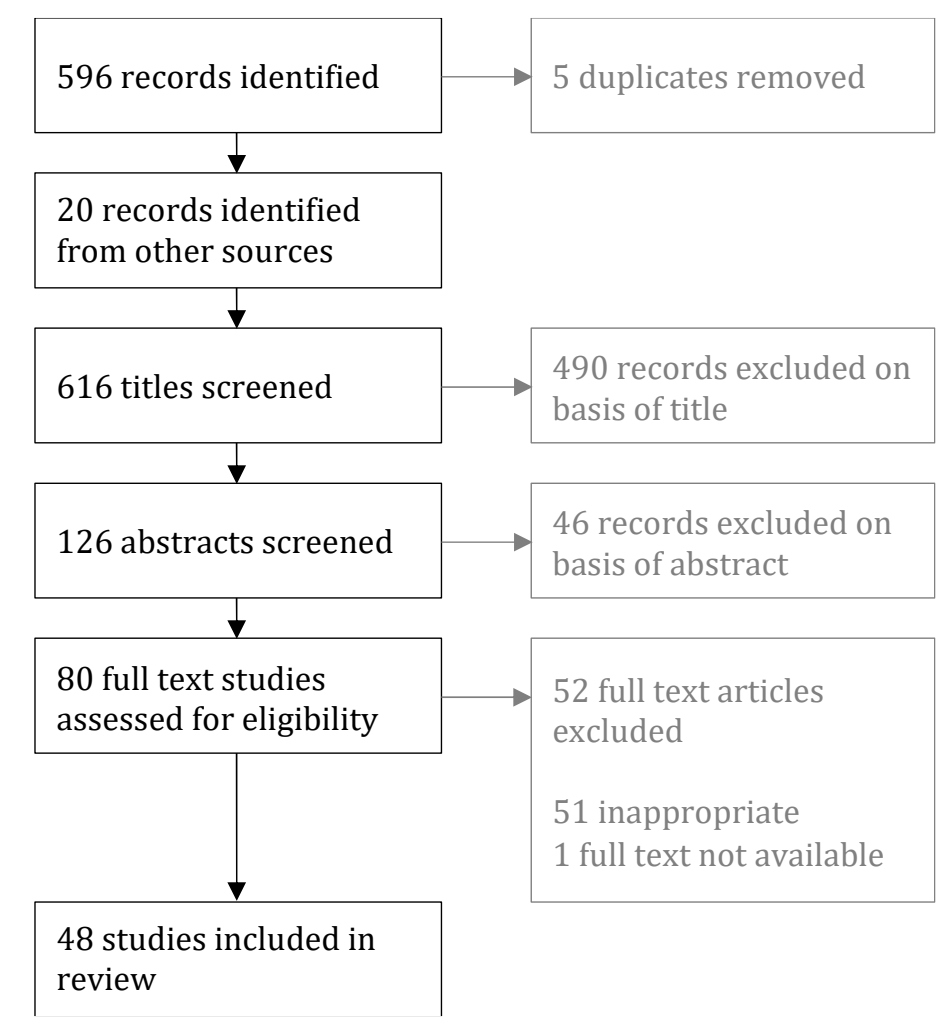

Of the 48 studies 31 were empirical (original research) and 15 non-empirical. Of the 31 empirical studies, 13 were experimental (6 RCTs, 3 pilot studies, 2 pre-post and 2 mixed methods) and 18 non-experimental ( 9 qualitative, 8 cross-sectionals and 1 technical paper). Of the non-empirical studies, there were 8 systematic reviews (including 2 meta-analyses and 1 systematic review of reviews), 1 realist review, one non-systematic review of reviews, two analytic reviews and 3 commentaries.

The 31 empirical studies took place in Australia (3), Belgium (1), Canada (2), Finland (1), Germany (1), Mexico (1), Netherlands (1), New Zealand (1), Sweden (1), Switzerland (2) U.K. (12) and USA (5). 4 studies were published since 2018 and 20 between 2015 and 2018, 9 between 2011 and 2014 and 13 before that with the earliest paper published in 1997. 
What are healthcare professionals currently doing?

Healthcare professional's perception of physical activity

The majority of healthcare professionals view physical activity as an important part of clinical care[28,29] and fundamental to the management of long-term conditions.[30] Primary care practitioners value physical activity as a non-medication option to promote self-management, particularly for overweight individuals and those living with health conditions and low mood.[31] Healthcare professionals are generally optimistic about physical activity[32] and view physical activity promotion positively,[29] although with less importance than other lifestyle factors such as smoking cessation.[33] Knowledge and perceived competence around physical activity conversations in clinical practice are variable, as is perceived success rates on changing patient behaviour.[28,32,33] This reflects a discrepancy between healthcare professionals' commitment to behaviour change in managing long-term conditions and their delivery of behaviour change support.[30] Where time is protected to facilitate physical activity conversations, it is valued,[34] but clinicians only follow strategies if they are convinced by their efficacy.[35]

\section{Healthcare professional knowledge and skill levels}

A lack of knowledge and skills around physical activity in clinical practice is widely reported,[12,23,29,32] reflecting a lack of adequate physical activity and behavioural change training.[36-42] Low self-efficacy and a lack of formal training are significant barriers to initiating physical activity conversations by healthcare professionals.[32,43] Encouragingly, education and skill development increases the frequency of physical activity conversations in routine practice and can help to standardise approaches.[29,32,43]

Healthcare professional confidence

Healthcare professionals with low confidence, who doubt their ability to have physical activity conversations that positively influence the behaviour of inactive people, talk about physical activity infrequently.[30,32,33,44] Confidence amongst healthcare professionals is lowest concerning specific rather than general advice on physical activity.[32,43] Healthcare professionals give general advice such as promoting national guidelines more frequently than they make an effort to engage and explore people's views on physical activity.[45] A qualitative exploration of the approach by healthcare professionals in primary care recorded two prevalent styles of communication that are detrimental to patient empowerment: diffidence and deflection. Diffident healthcare professionals tend to advise physical activity and behavioural change then withdraw from the topic before engaging with the individual's agenda. Deflective healthcare professionals gathered some information around physical activity then deferred discussion on the information to a future occasion that was not followed up. Both of these strategies may be worse than not raising the topic of physical activity. They leave people feeling responsible for their failure to live healthy, active lives but unclear on what to do about it or the role of healthcare in assisting them.[30]

Many General Practitioners (GPs) consider delivering physical activity information more frequently than they do. For example, an Australian study reported G.P.s might talk about physical activity in as few as $50 \%$ of the contacts they identify as likely to benefit from physical activity discussion.[43] This disparity is worst in some of the highest risk groups as conversations are observed less frequently in people living with health conditions as well as non-white ethnic groups and those without private health insurance in countries without state-delivered healthcare systems.[46] As well as 
physical activity conversations being undertaken less frequently in people with lower socioeconomic status also appear to be less effective.[29,47]

Where the individual skills of healthcare professionals are low, system support can be adequate. For instance, G.P.s with low self-efficacy around physical activity conversations view the opportunity to refer patients to a support counsellor favourably and will distribute information leaflets if made available.[28,31,32] Addressing this widespread deficit within healthcare will require a cohesive training programme to deliver physical activity support to people with health conditions successfully.[25] Achieving this will require a sustained, multifaceted approach as demonstrated by large scale centrally produced campaigns such as the U.K.'s 'Make Every Contact Count' campaign. Less than a third of healthcare professionals recognised the campaign to educate healthcare professionals around brief opportunistic intervention after three years.[48]

\section{What influences physical activity conversations in clinical practice? \\ Barriers to physical activity conversations}

With healthcare environments delivering a complex array of competing priorities, time is universally cited as the primary barrier to conversations on physical activity.[29,31$33,44,49]$ Time pressure is increased by associated problems such as staff shortage, higher priorities and general workload.[29,33] Lack of knowledge and training in behaviour change skills is a more prevalent barrier for healthcare professionals than physical activity and disease knowledge.[29,32,33,49,50] This widespread lack of training in behaviour change skills contributes to negative perceptions held by healthcare professionals, such as the fear of offending people and lack of conviction that they can influence physical activity behaviours successfully.[29,33,50,51]

\section{Safety considerations}

Although regular physical activity offers remarkable benefits to health and wellbeing, healthcare professionals and people living with medical conditions frequently lack confidence around the risk of adverse events. Poor reporting of adverse events in studies looking at physical activity in healthcare makes actual risks hard to quantify and does not help low confidence amongst healthcare professionals.[52] In a survey of 580 elderly participants, $77 \%$ recalled negative, no or contradicting advice about physical activity.[53] In keeping with advice from others, the authors concluded that warnings and constant reminders about potential risks might cause older people to become over-cautious about doing even moderate physical activities of daily life and eventually give up altogether.[53,54] Healthcare professionals should consider the impact of their advice on spouses and family members who frequently reinforce and can even augment negative messages around physical activity from healthcare professionals, further diminishing their self-efficacy and opportunities.[53] Physical activity schemes that address and support safety considerations improve attendance among older people supporting them around concerns such as cardiovascular adverse events, fear of falling and aggravating medical conditions.[55]

\section{Facilitators to physical activity conversations}

Limiting workload implications is critical for acceptability amongst healthcare professionals.[44] Integration into existing workstreams is beneficial for sustaining change in healthcare professional behaviour, and providing a structure on which to base physical activity conversations is valued by healthcare professionals.[32,56] Individual factors facilitating physical activity conversations include existing knowledge and skills and positive attitudes toward physical activity.[29] 
Healthcare professional characteristics

Positive attitudes toward physical activity may reflect personal behaviours as the personal activity levels of healthcare professionals strongly correlate with the frequency and quality of physical activity conversations they have, irrespective of their speciality or time in clinical practice.[12,33,44] Furthermore, patients themselves find recommendations from physically active healthcare professionals to be more compelling than equivalent advice from inactive healthcare professionals, demonstrating the impact of healthcare professional health and personal behaviours on how people interpret their advice.[12,50]

\section{What do people living with health conditions think? \\ Patient perception}

Most people attending healthcare appointments are interested in physical activity [44] and welcome behaviour change discussions with healthcare professionals, contrary to common perceptions.[50] For instance, healthcare professionals report a perceived lack of receptiveness to conversations on physical activity behaviour change as a major barrier.[51]

People living with medical conditions value a joined-up approach with integrated system support and find clear and consistent messaging around expectations helpful.[57] Patients recommend services deliver multidisciplinary support and appropriate referral in the understanding that different healthcare team members can contribute complementary support.[50,57] For instance, support from a doctor is a powerful enabler, but patients are realistic that they are unlikely to have the time and support longer conversations on physical activity.[50,57] In this circumstance, both distribution of tailored information and appropriate follow up are appreciated by patients.[58] Messages within these approaches are more successful if they highlight tangible benefits to the individual rather than focus on physical activity as a treatment for health problems.[59]

Three main themes emerged in a qualitative study looking at the patient perception of behaviour change conversations in primary care: 1. Behaviour change discussion is acceptable, particularly if it positively impacts health conditions and helps selfmanagement. 2. Good doctor-patient relationship forms a positive platform to facilitate behaviour change conversations. 3. Healthcare professionals, in general, are a credible source of information and well placed to offer behavioural change interventions.[50] Patients' perceived empowerment is a crucial determinant of success in exercise referral schemes and may form an influential target for intervention.[58]

\section{Person-centred approach}

Building perceived empowerment relies on skilful delivery of physical activity conversations, but clinicians seldom use empowering strategies when delivering brief advice.[60] Patients want tailored advice around physical activity, which relies on understanding individual circumstances and barriers. Established therapeutic relationships and increased knowledge of people's personal lives augment this process.[29,43,61]

Understanding the perspective of an individual is hard if they are denied the opportunity to talk. Observational studies record meagre contributions by patients to conversations on physical activity as healthcare professionals frequently do not offer 
them the chance to contribute to discussion around their behaviour.[60,62] For instance, people with pulmonary conditions are less likely to attend exercise referral appointments because of fear of worsening their shortness of breath, but people seldom get the opportunity to share concerns about this crucial barrier.[58]

Studies of practical behavioural change models, such as the '5As' approach,[63-65] document little use of the 'Agree' and 'Assist' stages, which is a problem as people are unlikely to engage in proactive behaviour change if the conversation focusses on 'Assessment' and unsolicited 'Advice'.[60,66] Patients recognise they may contribute to this problem, admitting that it is easy to respond defensively during behaviour change conversations.[50] To counter this, they recommend healthcare professionals avoid a 'preaching' style of communication favouring a person-tailored approach.[50] Being non-judgemental and building confidence are skills that patients value, and if not employed, repeated sessions of activity advice lead to resistance and irritation.[50,54] A realist review exploring factors contributing to the effectiveness of physical activity conversations in primary care concluded that, at a minimum, physical activity counselling should be based on the principles of motivational interviewing to anticipate barriers and discuss solutions.[25]

How does previous training impact conversations?

Old habits die hard

Traditional training and engrained consulting models make it hard for clinicians to change their consultation approach.[35] Healthcare professionals are typically trained to provide information and direction rather than to establish collaborative relationships with patients.[66] This approach may be particularly relevant for physicians who generally lead services geared toward delivering pharmaceutical treatment.[35] Defaulting and ascribing greater value to pharmaceutical options is increased when healthcare professionals hold negative attitudes towards physical activity.[67]

GPs feel that it takes time and skill to meet patients where they are to achieve a change in behaviour and report a lack of training in the relevant skills, which many perceive as too complicated to be practical.[35] Although disturbance to usual practice and routines is a potent barrier for healthcare professionals engaging in physical activity conversations, healthcare professionals recognise that alternative approaches, such as motivational interviewing, are likely to benefit their practice and patients.[34,35]

\section{Determinants of counselling behaviour}

Previous education and training on physical activity behaviour change skills are likely to be the most potent factor increasing the likelihood of healthcare professionals engaging patients in physical activity conversations.[68] However, behaviour change skills such as motivational interviewing have not been part of medical education, so there is a large skill deficit.[35] Encouragingly, healthcare professionals receive well designed post-graduate education positively and can find the training transformative in the way they approach conversations with their patients around behaviour change.[34,69]

The introduction of straightforward time-efficient protocols is well received and may be vital for supporting healthcare professionals with limited skills and 
experience.[70,71] No consistent relationship has been demonstrated between counselling practices between professional groups or years in practice, $[28,67]$ but conversations are observed more frequently during the management of particular medical conditions, including obesity, diabetes, hypertension and high cholesterol.[44,51,67] This represents an opportunity to capitalise on the inclination of healthcare professionals to talk more about physical activity to people living with these conditions and may also suggest that healthcare professionals fail to appreciate the breadth of their patients who may benefit from physical activity conversations.[44]

Successful education methods around physical activity conversations

A range of education strategies appears to positively influence healthcare professional conversations on physical activity, including presentations, practical workshops, and information delivery.[68,72] Feasible and acceptable approaches include motivational interviewing, physical activity screening tools, behaviour change techniques, multimodal approaches and consultation constructs such as the '5As' strategy.[34,68,73,74]

Healthcare professional engagement in education programmes can be improved when delivered by respected professionals or personal professional contacts and benefit from a transparent partnership with professional bodies.[44,72] training incorporating behavioural change approaches appears to change healthcare professional practice positively, but long-term behavioural change impact remains uncertain.[66] Studies report improved self-efficacy, longer and more frequent conversations on physical activity, enhanced perceived control and a more personcentred approach. $[34,66,68,73]$ However, educational interventions have not convincingly demonstrated a sustained change in the long term.[66]

On an individual level, whilst the intention to influence behaviour in consultation is essential, specific focus should be placed on strengthening the transition from intention [to raise the topic] to actual conversations.[17] Whilst improving the knowledge of physical activity recommendations and guidance is valuable, education materials on their own are unlikely to be effective in influencing behaviour, particularly if delivered passively.[29,30,82] The use of such materials is driven by their apparent utility as one of the cheapest behaviour change interventions available.[73,74] The use of written materials can be enhanced if combined with active education strategies such as face to face teaching or supported group work as part of a multifaceted education strategy.[73,83] Approaches can be further enhanced when tailored to the target group, such as focusing on particular disease areas.[84] Delivering education with tools to support assessment and the delivery of individualised advice enhances intervention effectiveness,[82] as does focussed work on message dissemination.[85]

\section{What structure should a good conversation follow?}

Structure

Physical activity screening tools can provide a positive prompt for physical activity conversations.[44] Various self-report tools exist, including the 'physical activity vital sign', a two-step screening question successfully integrated into large healthcare systems.[15] Consideration should be given to the timing of screening questions in healthcare, particularly for chronic disease management; for instance, patients consider it unacceptable if done more than twice annually.[44] Data derived from routine screening forms a vital part of developing an understanding of 'what works' around physical activity interventions. At a minimum, data capture should include the percentage of people meeting national physical activity recommendations.[73] 
A range of conceptual frameworks support the delivery of brief advice, but it is not clear what is most effective or the best fit for clinical practice.[75]. NICE advocates using the 5 A's model (Ask, Advise, Assess, Assist, Arrange) [63-65] whilst others have developed variants specific to physical activity, such as the Ask Advise Assist model for cancer care.[76,77]

Critics of the ' 5 A's, 'FRAMES' and similar assessment-focused intervention models argue that early screening and a focus on control theory lead to premature, cliniciandriven planning, omitting critical steps in helping an individual move toward sustainable behaviour change.[54] Given the range of non-physiological barriers to physical activity that exist, authors suggest that conversations must offer more than simply advising patients to increase physical activity.[25] Implementation trials incorporating conceptual frameworks demonstrate a range of limitations. Before and after training in the 5 A's structure, healthcare professionals predominantly use the Ask and Advise steps, reflecting a long-established transactional consultation method.[28,60,78] With a reliance on these steps, General Practitioners default to deferral strategies. When they recognise physical activity is important, they will tell the individual they need to be more active and then close the conversation.[30,35] Patient questions rarely emerge in these discussions, which seldom consider their perspective.[62]

Although not enough on their own, interventions to build self-efficacy are vital determinants of successful strategies.[79-81] Motivational Interviewing is an effective and increasingly popular framework to support the development of self-efficacy and patient-led behaviour change in clinical practice.[82-84]

Time considerations are paramount when developing an intervention that successfully supports the demands of clinical practice.[15,48,51] For instance, in the UK, average GP consultations are 9.22 minutes, during which they must address a wide range of complex objectives.[50] On average, GPs apportion 55-90 seconds of this time to lifestyle conversation when they consider it relevant.[60,62] Consequently, some authors recommend that resource development focus on creating successful interventions lasting less than 1 minute,[49] whilst others recommend developing interventions with 5-minute targets. [47] Five-minute interventions may be more appropriate for healthcare professionals with more time available for physical activity conversations than GPs.

Healthcare professionals report finding training based on a structured approach acceptable and help them have effective conversations in less than 5 minutes.[71,85] Pedometer, motivation, action planning and diary interventions are feasible, acceptable and deliverable in primary care.[61] Pedometer (averaging 5 minutes) and motivational interventions (6.5 minutes) are cost-effective in primary care.[59] Healthcare professionals may favour pedometer interventions for their simplicity, although comparative long term impact on patient physical activity levels is unknown.[59] Across a heterogeneous range of physical activity interventions, the Number Needed to Treat (NNT) for a sedentary adult to meet physical activity recommendations at 12 months is 12 , with no difference observed between singlesession behavioural change advice or formal exercise referral.[52] 
What works when making change happen?

Balancing with other work pressures

An optimal solution likely permits a flexible approach to support clinicians in various environments and circumstances, and different roles. Addressing both the barrier of time and appropriate allocation of resources reflects NICE delivery recommendations of "very brief, brief, extended brief and high intensity" behavioural change interventions.[63]

An individual's primary reason for healthcare attendance alters healthcare professionals' perception of the importance of promoting physical activity.[29] For instance, primary care providers do not generally perceive physical activity conversations to be as important as other lifestyle factors such as smoking cessation.[33] Some healthcare professionals express a sense of inadequacy when influencing patients to increase physical activity and frustration that primary prevention takes a back seat due to service demands.[86] Consequently, balancing physical activity conversations with other clinical objectives and addressing knowledge and skill deficits is primarily important.[32]

\section{System delivery}

Identifying inactive adults and targeting them with physical activity interventions has the potential to provide significant health benefits.[87] However, blanket physical activity promotion and over-reliance on the impact of individual practitioner advice (particularly physicians) are ineffective strategies when employed in isolation.[54,66,88] Organisational change is crucial to sustaining behavioural change with a more significant impact than stand-alone individualised interventions.[89] Healthcare systems may need to undergo an extensive cultural shift to better support patients.[90] Organisational support placing value in physical activity interventions has a powerful impact on healthcare professional behaviour, particularly when coupled with education and training strategies. $[29,90]$ It may be that one of the reasons it can be hard to implement physical activity interventions is that, unlike pharmaceuticals, there is no established institutional support or pathway.[86]

Successful interventions need to be compatible with existing care pathways and systems.[56] An example is integrating physical activity advice with routine health checks, which is acceptable and feasible for healthcare professionals and patients alike.[61] Systems that encourage patients to prompt healthcare professionals to discuss physical activity may be more important than training healthcare professionals.[60] Patient initiation of physical activity conversations is a promising intervention target with the benefit of leading to more frequent conversations compromising in-depth discussion, and exploring individual values and agenda.[60]

Patient initiation of physical activity conversations can also promote a shared-care model, which is favoured by healthcare professionals, particularly when they are unclear about the effectiveness of physical activity advice.[35] Such collaborative models of care promoting joint responsibility for behaviour change will improve care but remain hard to achieve until shared care approaches become the foundation upon which healthcare systems set their targets.[30]

Care delivery is improved by developing clear referral pathways and developing local stakeholder networks supporting training, promotion, implementation and evaluation.[32] Pathways should be simple and benefit from peer support networks and intersectoral cooperation.[34,86,91] Pathway integration with non-healthcare 
physical activity and sport sector providers increases referral rates from healthcare professionals.[32,91] Professionals working in roles outside healthcare who have had adequate training in long term disease management and physical activity may form a vital part of successful physical activity integration. [69] Trust in third sector provider skills improves the confidence of healthcare professionals and people living with health conditions, helping them feel safe and secure in the face of concerns such as cardiovascular adverse events, fear of falling and aggravating medical conditions.[58,69]

\section{Financial considerations}

Interventions costing less than $£ 30,000$ per Quality of Life Year (QALY) are considered cost-effective to commission, and NICE estimate that the cost of QALY through a brief intervention in physical activity is somewhere between $£ 20$ and $£ 440$.[92] Physical activity interventions in clinical practice are therefore highly effective and costeffective when compared to usual care.[47,59,61,66,77,93-95]

For feasible physical activity interventions to be embedded in general healthcare services, appropriate reimbursement must be assured.[85] System reimbursement is required to ensure adequate prioritisation of physical activity initiatives,[51] which has a powerful impact on healthcare professional behaviour.[29]

Healthcare professionals referring people to schemes delivered by third sector providers need to consider individual financial implications as they can be costly, particularly to individuals from lower socioeconomic groups.[91] Skilful intermediaries such as social prescribers (UK) or community health workers (elsewhere) can help overcome socioeconomic barriers so add value to service integration. [69] However, schemes that reduce individual costs do not reliably demonstrate an uptake in participation, suggesting cost may be an 'excuse' rather than a primary barrier to behavioural change.[91]

\section{Healthcare professional roles}

Continuity of care helps people trust their healthcare professionals in conversations around behavioural change.[96] Healthcare professionals find that understanding their role in supporting people's journey to becoming more physically active is helpful.[97] Appreciation of their role in a system can help reduce the chances of healthcare professionals disengaging with behaviour change by deflecting conversations to another timepoint without a concrete plan.[30]

Often service expectation is left on healthcare professionals themselves to deliver physical activity interventions, but the opinion of healthcare professionals on their role in physical activity behaviour change is variable.[32,33,91] Integration of physical activity counsellors into care pathways can help save clinician time, impart physical activity and health knowledge that healthcare professionals may not have, and deliver good quality behavioural change support.[88,91,97] This approach reflects the structure of general care pathways, is favourably received by healthcare professionals and can help reduce ethnic and cultural barriers improving patient experience and engagement.[31,62,69,88]

\section{What interventions are successful}

Protocolising physical activity pathways can help improve delivery and organisational support, but keeping interventions simple maybe most important.[29,44,98] delivery appears to be efficacious when both face to face or through telehealth, with impact 
demonstrated across all healthcare professional groups.[88,99] Prompting strategies, either embedded in electronic medical systems or otherwise, can increase the frequency of conversations on physical activity in clinical practice.[29,98,100] Systems encouraging patients to prompt their clinicians can potentially improve the frequency and quality of physical activity conversations.[60] Also, automated patient-focussed prompt systems can help reinforce conversations with healthcare professionals and improve adherence with physical activity interventions, potentially offloading the demand of face to face contacts.[100]

Exercise prescription approaches can help to validate physical activity as a treatment option.[31] However, an exercise prescription approach is of limited value unless supported by clinician education and multifaceted intervention.[31,54,101,102] This may be due to distrust of physical activity prescription as some healthcare professionals feel the method lacks credibility and significance for the individual,[86] which is important since healthcare professionals will not engage in interventions they feel lack efficacy.[103]

Physical activity prescription, akin to the prescription of medications, encourage healthcare professionals to deliver direct advice, which is less likely to generate sustained behavioural change than person-centred conversational strategies.[54] Even single sessions delivered by clinicians or counselling specialists discussing patient motivation may increase physical activity at 12 months.[25]

Motivational interviewing is a proven technique to support person led behaviour change in clinical practice. Evidence supports a sustained increase in physical activity levels for people with health conditions that can be improved with follow up sessions.[83] It may be that very brief motivational based conversations can improve the likelihood of successful behavioural change by adding self-regulation strategies.[59] Walking interventions with or without technological support from pedometers or wearables support brief conversations clinical practice being achievable in just a few minutes and acceptable for most people, including the elderly.[34,56,61,95,100]. Trials comparing different intervention types demonstrate larger increases in physical activity through walking and motivational support than physical activity prescription, action planning, diary planning and goal setting, although all have a positive effect.[59,95] Identification of the appropriate strategy should be made in collaboration with the individual concerned.

\section{Useful outputs}

Environmental modification of the waiting room can help support physical activity behaviour change initiatives. Informational videos and leaflets made available in waiting rooms are approaches encouraged by patients.[43,96] Within consultations, prescription pads can be well received by clinicians[73] and it's encouraged to give supporting resources to patients at the end of conversations such as information booklets, workbooks and practical instruction.[29,34,68,73,98] Workbooks can be particularly helpful but are variably maintained without follow-up.[34] Trials suggest starting people with devices such as pedometers and wearables in clinic is feasible, acceptable, time and cost-efficient. $[47,59,73,87]$

Collaborative development of materials for patients to take away will likely improve their efficacy.[47] Workbooks providing a structured approach to planning coupled with instruction on how to perform an activity may improve confidence and feelings of control.[87,95] Walking interventions lend themselves well to this approach. 
A range of BCTs is identified as efficacious to support clinical conversations on physical activity.[61,96,104] They are listed in table 1 together with descriptions from BCT taxonomy v1.[105] Focusing training around successful BCTs shows promise.[34]

\section{Table 1. Promising behaviour change techniques from BCT taxonomy v7[105]}

\begin{tabular}{|c|c|c|}
\hline BCT & & $\begin{array}{l}\text { Description of BCT from BCT } \\
\text { taxonomy vl }\end{array}$ \\
\hline 1.1 & $\begin{array}{l}\text { Goal setting } \\
\text { (behaviour) }\end{array}$ & $\begin{array}{l}\text { Set or agree on a goal defined in } \\
\text { terms of the behaviour to be } \\
\text { achieved }\end{array}$ \\
\hline 1.2 & Problem solving & $\begin{array}{l}\text { Analyse, or prompt the person to } \\
\text { analyse, factors influencing the } \\
\text { behaviour and generate or select } \\
\text { strategies that include } \\
\text { overcoming barriers and/or } \\
\text { increasing facilitators (includes' } \\
\text { Relapse Prevention' and 'Coping } \\
\text { Planning') }\end{array}$ \\
\hline
\end{tabular}

\subsection{Action planning}

Prompt detailed planning of performance of the behaviour (must include at least one of context, frequency, duration and intensity). Context may be environmental (physical or social) or internal (physical, emotional or cognitive)

\section{Review behaviour goal(s) jointly} with the person and consider modifying goal(s) or behaviour change strategy in light of achievement. This may lead to resetting the same goal, a small change in that goal or setting a new goal instead of (or in addition to) the first, or no change

1.6 Discrepancy
between
current
behaviour and
goal

\subsection{Commitment}

Draw attention to discrepancies between a person's current behaviour (in terms of the form, frequency, duration, or intensity of that behaviour) and the person's previously set outcome goals, behavioral goals or action plans (goes beyond self-monitoring of behaviour)

Ask the person to affirm or reaffirm statements indicating commitment to change the behaviour

\section{Example of BCT}

Agree on a daily walking goal (e.g. 3 miles) with the person and reach agreement about the goal Set the goal of eating 5 pieces of fruit per day as specified in public health guidelines

Prompt the patient to identify barriers preventing them from starting a new exercise regime e.g., lack of motivation, and discuss ways in which they could help overcome them e.g., going to the gym with a buddy

\section{Prompt planning the performance of a} particular physical activity (e.g. running) at a particular time (e.g. before work) on certain days of the week

\section{Examine how well a person's} performance corresponds to agreed goals e.g. whether they walked an average of 7000 steps per day

Point out that the recorded exercise fell short of the goal set

$$
\begin{aligned}
& \text { Ask the person to use an "I will" } \\
& \text { statement to affirm or reaffirm a strong } \\
& \text { commitment (i.e. using the words } \\
& \text { "strongly", }
\end{aligned}
$$


2.2 Feedback on behaviour

Monitor and provide informative or evaluative feedback on performance of the behaviour (e.g. form, frequency, duration, intensity)

2.3 Self-monitoring of behaviour

\subsection{Social support} (unspecified)

4.1 Instruction on how to perform a behaviour

5.1 Information about health consequences
Establish a method for the person to monitor and record their behaviour(s) as part of a behaviour change strategy

Advise on, arrange or provide social support (e.g. from friends, relatives, colleagues,' buddies' or staff) or non-contingent praise or reward for performance of the behaviour. It includes encouragement and counselling, but only when it is directed at the behaviour (includes 'Motivational interviewing' and 'Cognitive Behavioral Therapy')

Advise or agree on how to perform the behaviour (includes' Skills training')

Provide information (e.g. written, verbal, visual) about health consequences of performing the behaviour "committed" or "high priority") to start, continue or restart their strengthening exercises

Review how many steps someone walked each day (as recorded on a pedometer)

Give patient a pedometer and a form for recording daily total number of steps

Give information about a peer support group that offers support for the behaviour
Advise the person how to use a cross trainer in the gym

Present the likelihood of worsening disability from knee osteoarthritis if activity levels are not gradually increased

\subsection{Information} about social and environmental consequences

5.4 Monitoring of emotional consequences

5.6 Information about emotional consequences
Provide information (e.g. written, verbal, visual) about social and environmental consequences of performing the behaviour Prompt assessment of feelings after attempts at performing the behaviour

\section{Provide information (e.g. written,} verbal, visual) about emotional consequences of performing the behaviour
Tell someone they are unlikely to be able to play with their grandchildren if they do not build strength

Agree that the person will record how they feel after taking their daily walk

Explain that regular physical activity increases happiness and life satisfaction

\subsection{Credible source}

\section{Present verbal or visual} communication from a credible source in favour of or against the behaviour
Present a speech given by a high status professional to emphasise the importance of not exposing patients to unnecessary radiation by ordering $x$ rays for back pain

Add benches to long hospital corridors to act as resting spots for inpatients who are building up their activity levels after illness

Prompt the person to tell themselves that a walk will be energising
15.4 Self-talk
Prompt positive self-talk (aloud or silently) before and during the behaviour 


\section{Discussion}

This scoping review provides a structured insight into current understanding of individual conversations on physical activity in healthcare and their relationship to system delivery. There is a reasonable breadth of evidence in the field, most of which has come from the last decade perhaps highlighting the recent recognition of the important of lifestyle interventions to improve population health and gradual shift toward preventative healthcare approaches.

There is clear evidence outlining the detrimental impact of physical inactivity on individual's health,[1,2] and this is reflected in the acceptability of interventions by clinicians in focussing on improving physical activity of patients.[28-31] In contrast to healthcare professional perceptions,[50] there is also agreement from patients that physical activity is an important area where involvement of healthcare professionals can have a positive impact,[44] particularly with a multidisciplinary approach[50,57]. Physical activity competes with a range of other priorities in clinical practice and is frequently less well-regarded than other lifestyle interventions, such as smoking cessation[33] despite evidence for physical inactivity contributing to more deaths per year than smoking.[7] This is particularly true when time pressure is factored in.[44] Lack of prioritisation may be due to a lack of education and awareness amongst healthcare professionals on the specific evidence highlighting the risks of physical inactivity.

One of the strongest predictors of clinician led conversations on physical activity is individual physical activity level, which may reflect an increased importance and understanding on the benefits of physical activity by active healthcare professionals.[12,29,33,44] As well as conversing frequently on physical activity, active healthcare professionals also shave more effective conversations irrespective of their formal training in behavioural change skills.[12,28,29,50] part of this discrepancy may be explained by self-directed efforts at training in an area healthcare professionals perceive to be important as currently, there is a significant gap in formal medical education for physical activity and behaviour change skills.[12,23,29,32,35,43,68] Those who do not actively seek to learn these skills about these interventions fail to benefit. In clinical practice, poor knowledge and skills amongst healthcare professionals is bourne in poor support for the most vulnerable members of society.[29,46,47] Amongst inactive and socio-economically disadvantaged individuals living with medical conditions conversations are often vague and limited $[32,43]$ which can be at best not beneficial, but at worst detrimental to the likelihood of an individual to further engage with efforts toward sustainable behaviour change.[30]

Whilst improvements in education offer great potential, consideration must also be made for strategies focussed on actions. For example NICE recommend the 5 A's approach, and there are variations of this which are also currently utilised in certain areas of healthcare.[63-65,76,106] However, there is a danger that the delivery of these approaches is skewed towards the components which give clinicians almost full control of the consultation.[28,30,35,54,60,62,66] In healthcare this makes the patient a passive recipient of instructions, $[60,62]$, which may reduce the likelihood of ongoing engagement as their ideas, concerns, and expectations are not considered.[29,43,58,61] Interventions biased toward healthcare professional input may also limit their inherent sustainability due to their reliance on the time and direct input of healthcare professionals at initial consultation and in follow-up. The exercise prescription model of care demands a high level of education and a multifaceted approach[31,54,101,102] and comes with a strong risk of stripping people of their autonomy.[54] With the 
dominance of didactic healthcare interventions such as pharmaceuticals in the current climate, alternative approaches must be considered to provide better support to inactive people through their interactions with healthcare professionals.[29,35,66]

Methods such as motivational interviewing offer a potential solution to this issue.[8284] Their efficacy is proven[25,83] and supported by clinicians[34,35] and patients alike.[58] By taking a non-judgemental, patient-centred approach from the outset they potentially reduce the dominance of the clinician's agenda in empowering people to find sustainable solutions.[25,50,54] In addition, by offering patients the locus of control, they have the potential to offload time-pressures on clinicians by encouraging patient self-regulation from the outset.[58,59] Patients are generally understanding of the time pressures on clinicians, and are supportive of adjunctive measures such as patient information leaflets and digital resources.[50,57]

Simple strategies improving patient control and responsibility, such as pedometers, have been shown to be feasible, acceptable, and time efficientinclinical practice. $[47,59,73,87]$ However, in isolation these interventions offer limited choice to the patient and do not address patient-specific concerns. A clear emphasis on individualised approach is required $[85,107]$ including taking into consideration the impact of specific diseases.[84] Specific education simplifying behaviour change skills like motivational interviewing is required, as healthcare professionals are under-skilled, and often view these approaches as unfeasibly complicated. $[35,68]$ Although education around behaviour change is beginning to improve in healthcare,[34,69] more needs to be done.[40,51,56,82] Well-developed tools can help reduce the education burden to help healthcare professionals deliver effective conversations around physical activity behavioural change.[70,71]

Organisational level leadership is fundamental in supporting effective person-centred care pathways.[54,66,88-90] Firstly, they can support empowering patient selfeducation with provision of resources such as leaflets, informational videos in waiting rooms, and support available for clinicians in consulting rooms.[29,34,43,68,73,96,108] A protocolised approach to clarify, and improve delivery and organisational support is also important $[29,44,98]$, and ensuring compatibility with current systems.[56,88,93,97] System-wide approaches should include non-healthcare stakeholders who play an essential role in the promotion and provision of physical activity.[32,69,109] The addition of physical activity practitioners such as community health workers helps integrate health and external sector initiative.[44,72] This is an effective strategy where confidence in physical activity conversations is low[28,31,32], and may offer an important initial step in improving physical activity support to people with health conditions. Finally, organisations must offer adequate reimbursement for physical activity interventions, as this has a powerful impact on healthcare professional behaviour.[29]

Healthcare led physical activity interventions are effective[47,59,61,66,93,94,110] and cost-effective[93] compared with usual care. Where time is precious[15,48,51,60,62], resources must be mindfully allocated to improve physical activity within a minute or a matter of minutes.[47,49,71,85] Patients play a critical role as prompters for discussion about physical activity and self-regulators in tracking their progress.[60,61] However, both the patient and healthcare professional must be invested in any intervention for it to be its most effective[32,33,35]. A shared-care model with organisational support is essential[35]. 


\section{Conclusions}

Physical activity conversations are an acceptable, effective, and cost-effective strategy to promote increasing physical activity amongst people living with health conditions. However, despite a growing body of evidence in support of its role, and in describing strategies to implement increased physical activity amongst patient populations, there remains significant scope for improvement. Future strategies must not only focus on improved education of healthcare professionals in the benefits on physical activity, but also in behavioural change and brief advice and intervention techniques which are patient-led rather than prescriptive. Such strategies must be conscious of the limited time available to the clinician and the patient, and nurture an environment in which both are invested in increasing a patient's physical activity. Such strategies would benefit from organisational support, involvement of established physical activity counsellors, and external physical activity stakeholders.

\section{References}

1 WHO. WHO | Global recommendations on physical activity for health. Geneva: : World Health Organization 2010.

http://www.who.int/dietphysicalactivity/publications/9789241599979/en/ (accessed 4 Aug 2015).

2 WHO. WHO | Global status report on noncommunicable diseases 2010. Published Online First: 2010.http://www.who.int/nmh/publications/ncd_report2010/en/ (accessed 19 Feb 2015).

3 Global Advocacy Council for Physical Activity International Society for Physical Activity and Health. The Toronto Charter for Physical Activity: A Global Call for Action. J Phys Act Health 2010;7 Suppl 3:S37085.http://www.ncbi.nlm.nih.gov/pubmed/21116016 (accessed 28 Nov 2017).

4 Global Advocacy for Physical Activity (GAPA) the Advocacy Council of the International Society for Physical Activity and Health (ISPAH). NCD prevention: investments [corrected] that work for physical activity. Br J Sports Med 2012;46:709-12. doi:10.1136/bjsm.2012.091485

5 ISPAH International Society for Physical Activity and Health IS for PA and $H$. The Bangkok Declaration on Physical Activity for Global Health and Sustainable Development. Br J Sports Med 2017;51:1389-91. doi:10.1136/bjsports-2017-098063

6 Brannan M, Bernardotto M, Clarke N, et al. Moving healthcare professionals - A whole system approach to embed physical activity in clinical practice. BMC Med Educ 2019;19:84. doi:10.1186/s12909-019-1517-y

7 Lee IM, Shiroma EJ, Lobelo F, et al. Effect of physical inactivity on major noncommunicable diseases worldwide: An analysis of burden of disease and life expectancy. Lancet 2012;380:219-29. doi:10.1016/S0140-6736(12)61031-9

8 Woodcock J, Franco OH, Orsini N, et al. Non-vigorous physical activity and allcause mortality: Systematic review and meta-analysis of cohort studies. Int J Epidemiol 2011;40:121-38. doi:10.1093/ije/dyq104

9 NICE. Behaviour change: individual approaches | Guidance and guidelines | NICE. Natl Inst Heal Care Excell 2014;PH 49.https://www.nice.org.uk/guidance/ph49 (accessed 15 Feb 2018).

10 NICE. Physical activity: brief advice for adults in primary care primary care. Natl Inst Heal care Excell Public Heal Guidel 44 2013;PH44.nice.org.uk/guidance/ph44

11 NICE. Physical activity : encouraging activity in all people in contact with the NHS. 2015.

12 Lobelo F, de Quevedo IG. The Evidence in Support of Physicians and Health Care Providers as Physical Activity Role Models. Am J Lifestyle Med

2014;10:1559827613520120-. doi:10.1177/1559827613520120 
Sassen B, Kok G, Vanhees L. Predictors of healthcare professionals' intention and behaviour to encourage physical activity in patients with cardiovascular risk factors. BMC Public Health 2011;11:246. doi:10.1186/1471-2458-11-246

14 Robinson JH, Callister LC, Berry JA, et al. Patient-centered care and adherence: Definitions and applications to improve outcomes. J. Am. Acad. Nurse Pract. 2008:20:600-7. doi:10.1111/j.1745-7599.2008.00360.x

15 Sallis R, Franklin B, Joy L, et al. Strategies for promoting physical activity in clinical practice. Prog Cardiovasc Dis 2015;57:375-86. doi:10.1016/j.pcad.2014.10.003

Dacey ML, Kennedy MA, Polak R, et al. Physical activity counseling in medical school education: a systematic review. Med Educ Online 2014;19:24325. doi:10.3402/MEO.V19.24325

17 Levy MD, Loy L, Zatz LY. Policy approach to nutrition and physical activity education in health care professional training. Am J Clin Nutr 2014;99:1194S-201S. doi:10.3945/ajcn.113.073544

18 Kordi R, Moghadam N, Rostami M. Sports and exercise medicine in undergraduate medical curricula in developing countries: a long path ahead. Med Educ Online 2011;16. doi:10.3402/meo.v16i0.5962

19 Joy EL, Blair SN, McBride P, et al. Physical activity counselling in sports medicine: a call to action. Br J Sports Med 2013;47:49-53. doi:10.1136/bjsports-2012-091620

20 Douglas F, Torrance N, van Teijlingen E, et al. Primary care staff's views and experiences related to routinely advising patients about physical activity. A questionnaire survey. BMC Public Health 2006;6:138. doi:10.1186/1471-2458-6-138

21 Knox ECL, Musson H, Adams EJ. Knowledge of physical activity recommendations in adults employed in England: associations with individual and workplacerelated predictors. Int J Behav Nutr Phys Act 2015;12:69. doi:10.1186/s12966-0150231-3

22 Knox ECL, Esliger DW, Biddle SJH, et al. Lack of knowledge of physical activity guidelines: can physical activity promotion campaigns do better? BMJ Open 2013;3:e003633. doi:10.1136/bmjopen-2013-003633

23 Chatterjee R, Chapman T, Brannan MG, et al. GPs' knowledge, use, and confidence in national physical activity and health guidelines and tools: a questionnaire-based survey of general practice in England. Br J Gen Pract 2017;67:e668-75. doi:10.3399/bjgp17X692513

24 Douglas F, van Teijlingen E, Torrance N, et al. Promoting physical activity in primary care settings: health visitors' and practice nurses' views and experiences. J Adv Nurs 2006;55:159-68. doi:10.1111/j.1365-2648.2006.03903.x

25 Gagliardi AR, Faulkner G, Ciliska D, et al. Factors contributing to the effectiveness of physical activity counselling in primary care: a realist systematic review. Patient Educ Couns 2015;98:412-9. doi:10.1016/j.pec.2014.11.020

26 Arksey H, O'Malley L. Scoping studies: Towards a methodological framework. Int J Soc Res Methodol Theory Pract 2005;8:19-32. doi:10.1080/1364557032000119616

27 Tricco AC, Lillie E, Zarin W, et al. PRISMA Extension for Scoping Reviews (PRISMAScR): Checklist and Explanation. https://doi.org/107326/M18-0850 2018;169:467-73. doi:10.7326/M18-0850

28 Diehl K, Mayer M, Mayer F, et al. Physical activity counseling by primary care physicians: attitudes, knowledge, implementation, and perceived success. J Phys Act Health 2015;12:216-23. doi:10.1123/jpah.2013-0273

29 Huijg JM, van der Zouwe N, Crone MR, et al. Factors Influencing Primary Health Care Professionals' Physical Activity Promotion Behaviors: A Systematic Review. Int J Behav Med 2015;22:32-50. doi:10.1007/s12529-014-9398-2

30 Hunter C, Chew-Graham CA, Langer S, et al. 'I wouldn't push that further because I don't want to lose her': A multiperspective qualitative study of behaviour change 
for long-term conditions in primary care. Heal Expect 2015;18:1995-2010. doi:10.1111/hex.12304

31 Patel A, Schofield GM, Kolt GS, et al. General practitioners' views and experiences of counselling for physical activity through the New Zealand Green Prescription program. BMC Fam Pract 2011;12:119. doi:10.1186/1471-2296-12-119

32 Albert FA, Crowe MJ, Malau-Aduli AEO, et al. Physical activity promotion: A systematic review of the perceptions of healthcare professionals. Int. J. Environ. Res. Public Health. 2020;17:1-36. doi:10.3390/ijerph17124358

33 Hebert ET, Caughy MO, Shuval K. Primary care providers' perceptions of physical activity counselling in a clinical setting: a systematic review. Br J Sports Med 2012;46:625-31. doi:10.1136/bjsports-2011-090734

34 Beighton C, Victor C, Normansell R, et al. 'It's not just about walking.....it's the practice nurse that makes it work': a qualitative exploration of the views of practice nurses delivering complex physical activity interventions in primary care. BMC Public Health 2015;15:1236. doi:10.1186/s12889-015-2568-6

35 Persson G, Brorsson A, Hansson EE, et al. Physical activity on prescription (PAP) from the general practitioner's perspective - a qualitative study. BMC Fam Pract 2013;14:1. doi:10.1186/1471-2296-14-128

36 Levy MD, Loy L, Zatz LY. Policy approach to nutrition and physical activity education in health care professional training. Am J Clin Nutr 2014;99:1194S-201S. doi:10.3945/ajcn.113.073544

37 Dacey ML, Kennedy MA, Polak R, et al. Physical activity counseling in medical school education: a systematic review. Med Educ Online 2014;19:24325.http://www.pubmedcentral.nih.gov/articlerender.fcgi?artid $=4111877$ \&tool=pmcentrez\&rendertype=abstract (accessed 24 Jun 2015).

38 Kordi R, Moghadam N, Rostami M. Sports and exercise medicine in undergraduate medical curricula in developing countries: a long path ahead. Med Educ Online 2011;16. doi:10.3402/meo.v16i0.5962

39 Weiler R, Chew S, Coombs N, et al. Physical activity education in the undergraduate curricula of all UK medical schools: are tomorrow's doctors equipped to follow clinical guidelines? Br J Sports Med 2012;46:1024-6. doi:10.1136/bjsports-2012-091380

40 Connaughton A V, Weiler RM, Connaughton DP. Graduating medical students' exercise prescription competence as perceived by deans and directors of medical education in the United States: implications for Healthy People 2010. Public Health Rep 2001;116:226-34. doi:10.1093/phr/116.3.226

41 Cardinal BJ, Park EA, Kim M, et al. If Exercise Is Medicine, Where Is Exercise in Medicine? Review of U.S. Medical Education Curricula for Physical ActivityRelated Content. J Phys Act Heal 2015;12:1336-43. doi:10.1123/jpah.2014-0316

42 Milton K, Larner J, Hanson S, et al. Embedding Physical Activity into the Healthcare Curriculum-A Case Study. Educ Prim Care 2020;31:176-9. doi:10.1080/14739879.2020.1744193

43 Bull FC, Schipper EC, Jamrozik K, et al. How can and do Australian doctors promote physical activity? Prev Med (Baltim) 1997;26:866-73. doi:10.1006/pmed.1997.0226

44 Allenspach EC, Handschin M, Kutlar Joss M, et al. Patient and physician acceptance of a campaign approach to promoting physical activity: the "Move for Health' project. Swiss Med Wkly 2007;137:292-9.

45 McKenna J, Vernon M. How general practitioners promote 'lifestyle' physical activity. Patient Educ Couns 2004;54:101-6. doi:10.1016/S0738-3991(03)00192-7

46 Heaton PC, Frede SM. Patients' need for more counseling on diet, exercise, and smoking cessation: results from the National Ambulatory Medical Care Survey. J 
Am Pharm Assoc (2003) 2006;46:364-9. doi:10.1331/154434506777069516

47 Lamming L, Pears S, Mason D, et al. What do we know about brief interventions for physical activity that could be delivered in primary care consultations? A systematic review of reviews. Prev Med (Baltim) 2017;99:152-63. doi:10.1016/J.YPMED.2017.02.017

48 Keyworth C, Epton T, Goldthorpe J, et al. Are healthcare professionals delivering opportunistic behaviour change interventions? A multi-professional survey of engagement with public health policy. Implement Sci 2018;13:122. doi:10.1186/s13012-018-0814-x

49 Eakin E, Brown W, Schofield G, et al. General practitioner advice on physical activity--who gets it? Am J Health Promot 2007;21:225-8. doi:10.4278/0890-117121.4 .225

50 Keyworth C, Epton T, Goldthorpe J, et al. Perceptions of receiving behaviour change interventions from GPs during routine consultations: A qualitative study. PLoS One 2020;15. doi:10.1371/journal.pone.0233399

51 Eakin EG, Smith BJ, Bauman AE. Evaluating the Population Health Impact of Physical Activity Interventions in Primary Care-Are We Asking the Right Questions? J Phys Act Heal 2005;2:197-215. doi:10.1123/jpah.2.2.197

52 Orrow G, Kinmonth A-L, Sanderson S, et al. Effectiveness of physical activity promotion based in primary care: systematic review and meta-analysis of randomised controlled trials. BMJ 2012;344:e1389. doi:10.1136/bmj.e1389

53 Hirvensalo M, Heikkinen E, Lintunen T, et al. Recommendations for and warnings against physical activity given to older people by health care professionals. Prev Med (Baltim) 2005;41:342-7. doi:10.1016/j.ypmed.2004.11.020

54 Hillsdon M, Thorogood M, White I, et al. Advising people to take more exercise is ineffective: A randomized controlled trial of physical activity promotion in primary care. Int J Epidemiol 2002;31:808-15. doi:10.1093/ije/31.4.808

55 Mills $\mathrm{H}$, Crone D, James DVB, et al. Exploring the perceptions of success in an exercise referral scheme: a mixed method investigation. Eval Rev 2012;36:407-29. doi:10.1177/0193841X12474452

56 Cooke AB, Pace R, Chan D, et al. A qualitative evaluation of a physician-delivered pedometer-based step count prescription strategy with insight from participants and treating physicians. Diabetes Res Clin Pract 2018;139:314-22. doi:10.1016/j.diabres.2018.03.008

57 Birtwistle SB, Ashcroft G, Murphy R, et al. Factors influencing patient uptake of an exercise referral scheme: a qualitative study. Health Educ Res 2019;34:113-27. doi:10.1093/her/cyy038

58 Mills $\mathrm{H}$, Crone D, James DVB, et al. Exploring the perceptions of success in an exercise referral scheme: a mixed method investigation. Eval Rev 2012;36:407-29. doi:10.1177/0193841X12474452

59 Pears S, Bijker M, Morton K, et al. A randomised controlled trial of three very brief interventions for physical activity in primary care. BMC Public Health 2016;16:1033. doi:10.1186/s12889-016-3684-7

60 Carroll JK, Fiscella K, Meldrum SC, et al. Clinician-patient communication about physical activity in an underserved population. J Am Board Fam Med 2008;21:11827. doi:10.3122/jabfm.2008.02.070117

61 Pears S, Morton K, Bijker M, et al. Development and feasibility study of very brief interventions for physical activity in primary care. BMC Public Health 2015;15:333. doi:10.1186/s12889-015-1703-8

62 Bardach SH, Schoenberg NE. The content of diet and physical activity consultations with older adults in primary care. Patient Educ Couns 2014;95:31924. doi:10.1016/j.pec.2014.03.020 
63 NICE. Behaviour change: individual approaches | Guidance and guidelines | NICE. Natl Inst Heal Care Excell 2014;:PH 49.

64 NICE. Behaviour change: general approaches PH6. London: 2007.

65 NICE. Physical activity: brief advice for adults in primary care primary care. Natl Inst Heal care Excell Public Heal Guidel 44 2013;PH44.

66 Galaviz KI, Estabrooks PA, Ulloa EJ, et al. Evaluating the effectiveness of physician counseling to promote physical activity in Mexico: an effectiveness-

implementation hybrid study. Transl Behav Med 2017;7:731-40. doi:10.1007/s13142017-0524-y

67 Huijg JM, van der Zouwe N, Crone MR, et al. Factors influencing the introduction of physical activity interventions in primary health care: a qualitative study. Int J Behav Med 2015;22:404-14. doi:10.1007/s12529-014-9411-9

68 Windt J, Windt A, Davis J, et al. Can a 3-hour educational workshop and the provision of practical tools encourage family physicians to prescribe physical activity as medicine? A pre-post study. BMJ Open 2015;5. doi:10.1136/bmjopen2015-007920

69 Costa EF, Guerra PH, Santos TI Dos, et al. Systematic review of physical activity promotion by community health workers. Prev Med (Baltim) 2015;81:114-21. doi:10.1016/j.ypmed.2015.08.007

70 Eakin EG, Brown WJ, Marshall AL, et al. Physical activity promotion in primary care: bridging the gap between research and practice. Am J Prev Med 2004;27:297-303. doi:10.1016/j.amepre.2004.07.012

71 Houde SC, Melillo KD. Physical activity and exercise counseling in primary care. Nurse Pract 2000;25:8,11-14,17-19. doi:10.1097/00006205-200025080-00001

72 Brannan M, Bernardotto M, Clarke N, et al. Moving healthcare professionals - a whole system approach to embed physical activity in clinical practice. BMC Med Educ 2019;19. doi:10.1186/s12909-019-1517-y

73 Fowles JR, O'Brien MW, Solmundson K, et al. Exercise is Medicine Canada physical activity counselling and exercise prescription training improves counselling, prescription, and referral practices among physicians across Canada. Appl Physiol Nutr Metab 2018;43:535-9. doi:10.1139/apnm-2017-0763

74 Gallegos-Carrillo K, García-Peña C, Salmerón J, et al. Brief Counseling and Exercise Referral Scheme: A Pragmatic Trial in Mexico. Am J Prev Med 2017;52:249-59. doi:10.1016/j.amepre.2016.10.021

75 Michie S, van Stralen MM, West R. The behaviour change wheel: a new method for characterising and designing behaviour change interventions. Implement Sci 2011;6:42. doi:10.1186/1748-5908-6-42

76 Campbell A, Foster J, Stevinson C, et al. Macmillan: Promote Physical Activity. 2012.

77 Webb J, Foster J, Poulter E. Increasing the frequency of physical activity very brief advice for cancer patients. Development of an intervention using the behaviour change wheel. Public Health 2016;133:45-56. doi:10.1016/j.puhe.2015.12.009

78 McKenna J, Naylor PJ, McDowell N. Barriers to physical activity promotion by general practitioners and practice nurses. Br J Sports Med 1998;32:2427.http://www.ncbi.nlm.nih.gov/pubmed/9773175 (accessed 22 Aug 2018).

79 Olander EK, Fletcher H, Williams S, et al. What are the most effective techniques in changing obese individuals' physical activity self-efficacy and behaviour: a systematic review and meta-analysis. Int J Behav Nutr Phys Act 2013;10.

80 Bauman AE, Reis RS, Sallis JF, et al. Correlates of physical activity: why are some people physically active and others not? Lancet (London, England) 2012;380:25871. doi:10.1016/S0140-6736(12)60735-1

81 Knittle K, Nurmi J, Crutzen R, et al. How can interventions increase motivation for 
physical activity? A systematic review and meta-analysis. Health Psychol Rev 2018;:1-20. doi:10.1080/17437199.2018.1435299

82 Rubak S, Sandbaek A, Lauritzen T, et al. Motivational interviewing: a systematic review and meta-analysis. Br J Gen Pract 2005;55:305-12.

83 O'Halloran PD, Blackstock F, Shields N, et al. Motivational interviewing to increase physical activity in people with chronic health conditions: A systematic review and meta-analysis. Clin Rehabil 2014;28. doi:10.1177/0269215514536210

84 Morton K, Beauchamp M, Prothero A, et al. The effectiveness of motivational interviewing for health behaviour change in primary care settings: a systematic review. Health Psychol Rev 2015;9. doi:10.1080/17437199.2014.882006

85 Märki A, Bauer GB, Angst F, et al. Systematic counselling by general practitioners for promoting physical activity in elderly patients: a feasibility study. Swiss Med Wkly 2006;136:482-8.

86 Persson G, Brorsson A, Hansson EE, et al. Physical activity on prescription (PAP) from the general practitioner's perspective - a qualitative study. BMC Fam Pract 2013;14. doi:10.1186/1471-2296-14-128

87 Howlett N, Trivedi D, Troop NA, et al. Are physical activity interventions for healthy inactive adults effective in promoting behavior change and maintenance, and which behavior change techniques are effective? A systematic review and metaanalysis. Trans/ Behav Med Published Online First: 2018. doi:10.1093/tbm/iby010

88 Tulloch $\mathrm{H}$, Fortier M, Hogg W. Physical activity counseling in primary care: who has and who should be counseling? Patient Educ Couns 2006;64:6-20. doi:10.1016/j.pec.2005.10.010

89 Robertson R, Jochelson K. Interventions that change clinician behaviour: Mapping the literature. King's Fund, London 2006.

90 Hunter RF, Tully MA, Donnelly P, et al. Knowledge of UK physical activity guidelines: Implications for better targeted health promotion. Prev Med (Baltim) 2014;65:33-9. doi:10.1016/j.ypmed.2014.04.016

91 Leemrijse CJ, de Bakker DH, Ooms L, et al. Collaboration of general practitioners and exercise providers in promotion of physical activity a written survey among general practitioners. BMC Fam Pract 2015;16:96. doi:10.1186/s12875-015-0316-8

92 NICE. Four commonly used methods to increase physical activity. UK: : NICE Guidance 2015.

93 GC V, Wilson ECF, Suhrcke M, et al. Are brief interventions to increase physical activity cost-effective? A systematic review. Br. J. Sports Med. 2016;50:408-17. doi:10.1136/bjsports-2015-094655

94 Stead LF, Buitrago D, Preciado N, et al. Physician advice for smoking cessation. In: Stead LF, ed. Cochrane Database of Systematic Reviews. Chichester, UK: : John Wiley \& Sons, Ltd 2013. CD000165. doi:10.1002/14651858.CD000165.pub4

95 Van Hoecke A-S, Delecluse C, Bogaerts A, et al. The long-term effectiveness of need-supportive physical activity counseling compared with a standard referral in sedentary older adults. J Aging Phys Act 2014;22:186-98. doi:10.1123/japa.20120261

96 Keyworth C, Epton T, Goldthorpe J, et al. Delivering Opportunistic Behavior Change Interventions: a Systematic Review of Systematic Reviews. Prev Sci 2020;21:319-31. doi:10.1007/s11121-020-01087-6

97 Berra K, Rippe J, Manson JE. Making Physical Activity Counseling a Priority in Clinical Practice. JAMA 2015;314:1. doi:10.1001/jama.2015.16244

98 Ackermann RT, Deyo RA, LoGerfo JP. Prompting primary providers to increase community exercise referrals for older adults: a randomized trial. J Am Geriatr Soc 2005;53:283-9. doi:10.1111/j.1532-5415.2005.53115.x

James EL, Ewald B, Johnson N, et al. Efficacy of GP referral of insufficiently active 
patients for expert physical activity counseling: protocol for a pragmatic randomized trial (The NewCOACH trial). BMC Fam Pract 2014;15:218.

doi:10.1186/s12875-014-0218-1

100 Dubbert PM, Cooper KM, Kirchner KA, et al. Effects of nurse counseling on walking for exercise in elderly primary care patients. J Gerontol A Biol Sci Med Sci 2002;57:M733-40. doi:10.1093/gerona/57.11.m733

101 Sims J. The Victorian Active Script Programme: promising signs for general practitioners, population health, and the promotion of physical activity. $\mathrm{Br} \mathrm{J}$ Sports Med 2004;38:19-25. doi:10.1136/bjsm.2002.001297

102 Grandes G, Sanchez A, Sanchez-Pinilla RO, et al. Effectiveness of physical activity advice and prescription by physicians in routine primary care: a cluster randomized trial. Arch Intern Med 2009;169:694-701.

doi:10.1001/archinternmed.2009.23

103 Bélanger M, Phillips EW, O’Rielly C, et al. Longitudinal qualitative study describing family physicians' experiences with attempting to integrate physical activity prescriptions in their practice: 'It's not easy to change habits'. BMJ Open 2017;7. doi:10.1136/bmjopen-2017-017265

104 Webb J, Hall J, Hall K, et al. Increasing the frequency of physical activity very brief advice by nurses to cancer patients. A mixed methods feasibility study of a training intervention. Public Health 2016;139:121-33. doi:10.1016/j.puhe.2016.05.015

105 Michie S, Richardson M, Johnston M, et al. The behavior change technique taxonomy (V1) of 93 hierarchically clustered techniques: Building an international consensus for the reporting of behavior change interventions. Ann Behav Med 2013;46:81-95. doi:10.1007/s12160-013-9486-6

106 Webb J, Hall J, Hall K, et al. Increasing the frequency of physical activity very brief advice by nurses to cancer patients. A mixed methods feasibility study of a training intervention. Public Health 2016;139:121-33. doi:10.1016/j.puhe.2016.05.015

107 Rubak S, Sandbaek A, Lauritzen T, et al. Motivational interviewing: a systematic review and meta-analysis. Br J Gen Pract 2005;55:30512.http://www.ncbi.nlm.nih.gov/pubmed/15826439 (accessed 17 Apr 2018).

108 Ackermann RT, Deyo RA, LoGerfo JP. Prompting Primary Providers to Increase Community Exercise Referrals for Older Adults: A Randomized Trial. J Am Geriatr Soc 2005;53:283-9. doi:10.1111/j.1532-5415.2005.53115.x

109 Leemrijse CJ, de Bakker DH, Ooms L, et al. Collaboration of general practitioners and exercise providers in promotion of physical activity a written survey among general practitioners. BMC Fam Pract 2015;16:96. doi:10.1186/s12875-015-0316-8

110 Webb J, Foster J, Poulter E. Increasing the frequency of physical activity very brief advice for cancer patients. Development of an intervention using the behaviour change wheel. Public Health 2016;133:45-56. doi:10.1016/j.puhe.2015.12.009 